\title{
Effect of Comprehensive Nursing on Cervical Cancer Patients
}

\author{
Xiaoyun Liu* \\ Weihai Central Hospital, Weihai 264400, Shandong Province, China \\ *Corresponding author: Xiaoyun Liu, xt666999@yeah.net
}

\begin{abstract}
Objective: To explore the effect of comprehensive nursing on patients with cervical cancer. Methods: A total of 100 cervical cancer patients, treated in Weihai Central Hospital from May 2020 to May 2021, were included in this study. The patients were cared for by different methods. They were divided into two groups, the control group and the experimental group, in which routine nursing and comprehensive nursing were given, respectively. The effect of the two different nursing methods on the patients were compared and analyzed. Results: After nursing intervention, the incidence of complications and the scores of depression and anxiety of the experimental group were significantly lower compared with the control group, $P$ $<0.05$. Conclusion: The application of comprehensive nursing among patients with cervical cancer has a positive significance on their recovery. It reduces the incidence of adverse reactions, improve patients' negative emotions, and improve their satisfaction.
\end{abstract}

Keywords: Comprehensive nursing; Patients with cervical cancer; Nursing effect; Adverse reaction

Publication date: November 2021; Online publication: November 30, 2021

\section{Introduction}

As far as cervical cancer is concerned, it is a common disease in women but a major disease that poses a serious threat to women's life and health. The most common type is squamous cell carcinoma ${ }^{[1]}$. It usually occurs at the junction of cervical squamous cells and columnar cells. In recent years, the incidence rate of cervical cancer has been increasing. It has caused serious threat to people's life and health. The treatment of these patients is mainly based on surgical treatment, radiotherapy, and chemotherapy. In the treatment process, effective nursing intervention is also required to ensure a better prognosis ${ }^{[2]}$. Therefore, comprehensive nursing was applied in this study to explore the impact of this nursing intervention on patients through comparative analysis.

\section{Materials and methods}

\subsection{General information}

This study was conducted in Weihai Central Hospital. A total of 100 cervical cancer patients who received treatment from May 2020 to May 2021 were included in this study, all of which were women. The oldest was 67 years old and the youngest was 32 years old, with an average age of $53.23 \pm 3.23$. Among them, there were 24 cases with stage IIA cancer, 28 cases with stage IIB cancer, 10 cases with stage IIIA cancer, 18 cases with stage IIIB cancer, and 20 cases with stage IV cancer. Among the patients, the largest tumor diameter was $5 \mathrm{~cm}$ and the smallest was $2 \mathrm{~cm}$; the average tumor diameter was $3.23 \pm 3.23 \mathrm{~cm}$. This study was carried out in a controlled manner. The patients were divided into two groups, the control group and the experimental group, with 50 patients in each group. Different nursing methods were used for each group. 
The general data of the two groups were compared using statistical methods, showing $P>0.05$. This indicates that a controlled experiment was established.

The inclusion criteria were patients with cervical cancer, patients who received percutaneous arterial chemotherapy drug infusion and embolization, as well as patients who were informed of the study and agreed to participate. The study was approved by the hospital's ethics committee.

The exclusion criteria were patients with poor compliance and those who refused to cooperate with the study, patients with other organ diseases, patients with mental abnormalities, or patients with impaired consciousness.

\subsection{Methods}

The patients in the control group received routine nursing, which includes observing their condition, providing symptomatic treatment, strictly following the principle of aseptic operation, avoiding infection, etc.

The patients in the experimental group received comprehensive nursing. In the implementation of comprehensive nursing, psychological nursing was first initiated. Most cervical cancer patients are worried about their condition and have heavy psychological burden. This may lead to complications, such as depression, fear, and other negative emotions; in addition, it would have a negative impact on the treatment compliance. In consideration of the actual situation of each patient, the nursing staffs would then try to understand the psychological changes in each patient, strengthen communication with each patient, carry out psychological counseling, guide the patients to release their emotions appropriately, and encourage the patients to talk, so as to reduce the psychological pressure and improve their negative emotions. This process can be applied in combination with various methods, including music therapy and static sitting. Secondly, the patients received health education, in order to improve their awareness, establish their selfconfidence, improve their attention, and prevent complications from occurring by explaining the treatment methods, possible clinical effects, complications, and prevention methods. At the same time, diet management was strengthened for these patients. Active cooperation was warranted from the patients in this process and their feelings were taken into consideration. The impact of diet on their condition was explained, reasonable diet plans were formulated for the patients in consideration of their conditions and daily preferences, the intake of high calorie, high protein, low salt, and digestible food was encouraged, the patients were encouraged to eat less and more often with more fresh vegetables and fruits, and they were supplemented with vitamins as well as prohibited from eating stimulating, spicy, raw, and cold food to help them develop good eating habits. Post-operative care was then given to the patients, whereby the ward environment was managed well by regular cleaning, disinfecting, and sterilizing, as well as adjusting the indoor temperature and humidity to improve the comfort of patients, in order to avoid the adverse impact of the environment on patients. Social support was also given to the patients. Most patients with cervical cancer suffer from intense psychological pressure, which would affect their social life. The nurses had to actively encourage the patients, promote more exchanges with their families, and arrange exchange meetings in order to promote mutual understanding among patients and encourage patients to share with one another, so as to promote their social ability and improve the discomfort faced by the patients. Finally, the patients were given venipuncture care. The principle of a sterile procedure was strictly implemented in this process. The nurses should continuously improve their venipuncture skills, ensure the success of a onetime puncture, reduce the pain of repeated punctures, reduce the probability of lower limb venipuncture, and avoid phlebitis in patients, so as to improve the quality of life of patients. 


\subsection{Observation indexes}

The Self-Rating Depression Scale and the Self-Rating Anxiety Scale were used to evaluate the emotions of the patients in both groups. The cut-off point for the former is 53, whereas that of the latter is 50. If the score of the patient is more than 53 or 50 points, it means that the patient has depression or anxiety, respectively. The higher the score, the more serious the depression or anxiety.

The incidence of complications of both groups were compared. The complications mainly included gastrointestinal reaction, reduction of leukocyte count, and bone marrow suppression.

\subsection{Statistical analysis}

SPSS 20.0 software was used to evaluate the data. For the calculation of measurement data, the results were mainly in $\bar{x} \pm \mathrm{s}$, and the verification was expressed by $\mathrm{t}$ value. For the counting data, the comparison results were counted by $\mathrm{n} / \%$, and the verification was expressed by $\chi^{2}$ value. $P<0.05$ indicates a significant difference in the results.

\section{Results}

\subsection{Emotion scores}

There was no significant difference in the scores of depression and anxiety between the control group and the experimental group before nursing, $P>0.05$. However, after nursing, the scores changed. In contrast, the scores of depression and anxiety of the experimental group were lower, which was significantly different from the control group, $P<0.05$ (Table 1).

Table 1. Comparison of depression and anxiety scores between the two groups before and after nursing $(\bar{x} \pm \mathrm{s})$

\begin{tabular}{lcccc}
\hline \multirow{2}{*}{ Group } & \multicolumn{2}{c}{ Depression (score) } & \multicolumn{2}{c}{ Anxiety (score) } \\
\cline { 2 - 5 } & Before nursing & After nursing & Before nursing & After nursing \\
\hline Experimental group $(\mathrm{n}=50)$ & $53.45 \pm 5.44$ & $37.45 \pm 5.23$ & $57.44 \pm 7.11$ & $36.56 \pm 5.44$ \\
Control group $(\mathrm{n}=50)$ & $53.95 \pm 5.20$ & $46.56 \pm 5.49$ & $57.05 \pm 7.39$ & $44.67 \pm 5.38$ \\
$\mathrm{t}$ & 0.395 & 6.596 & 0.945 & 8.004 \\
$P$ & $>0.05$ & $<0.05$ & $>0.05$ & $<0.05$ \\
\hline
\end{tabular}

\subsection{Incidence of complications}

The patients in both groups developed complications, but there was a significant difference in the incidence between the two groups $(P<0.05)$. The incidence of complications of the experimental group was lower compared to the control group (Table 2).

Table 2. Comparison of the incidence of complications between the two groups (n/\%)

\begin{tabular}{lcccc}
\hline Group & Gastrointestinal reaction & Reduced leukocyte count & Myelosuppression & Incidence rate \\
\hline Experimental group $(\mathrm{n}=50)$ & $1(2.00)$ & $1(2.00)$ & $1(2.00)$ & $3(6.00)$ \\
Control group $(\mathrm{n}=50)$ & $3(6.00)$ & $3(6.00)$ & $5(10.00)$ & $11(22.00)$ \\
$\mathrm{X}^{2}$ & & & & 6.596 \\
$P$ & & & & $<0.05$ \\
\hline
\end{tabular}




\section{Discussion}

In recent years, the incidence of cervical cancer has been showing an increasing trend ${ }^{[3]}$. The clinical treatment for these patients is mainly through surgical methods, radiotherapy, and chemotherapy. These interventions would not completely cure the disease, but only prolong the life of patients ${ }^{[4]}$. At the same time, these interventions may bring great damage to the patients; the tendency to develop negative emotions may increase among these patients along with the risk of developing adverse effects. Therefore, it is necessary to pay more attention to this condition and offer patients more effective nursing interventions ${ }^{[5]}$. This study mainly applied the comprehensive nursing method to effectively consider the patients in their situation in a comprehensive manner ${ }^{[6]}$. Comprehensive intervention includes psychological nursing, health education, dietary nursing, post-operative nursing, venipuncture nursing, etc. It ensures that when patients develop adverse reactions, positive treatment can be provided in a timely manner. Improving patients' negative emotions, compliance, treatment environment, and their understanding of their own diseases as well as establishing their self-confidence are of positive significance to improving their quality of life and reducing the incidence of complications ${ }^{[1]}$. The results obtained from this study showed that the scores of depression and anxiety as well as the incidence of complications of the experimental group were significantly lower compared to those of the control group. This shows that the clinical effect of comprehensive nursing is remarkable.

In conclusion, comprehensive nursing in patients with cervical cancer provides a significant effect. It reduces the incidence of adverse reactions and improves patients' negative emotions. It is of great significance for clinical development and should be popularized.

\section{Disclosure statement}

The author declares that there is no conflict of interest.

\section{References}

[1] Sui X, Zhang S, Zhai C, et al., 2019, Discussion on Application Effect of Comprehensive Nursing Intervention in Patients with Cervical Cancer Undergoing Radiotherapy. Systems Medicine, 004(003): 165-167.

[2] Wang S, Li H, 2019, Clinical Application Effect of Comprehensive Nursing Intervention in Patients with Cervical Cancer after Radiotherapy. Journal of Practical Gynecologic Endocrinology (Electronic Edition), (20): 186.

[3] Chen Q, 2019, Study on the Effect of Comprehensive Nursing Intervention on Alleviating Patients' Anxiety After Radical Resection of Cervical Cancer. Dietary Health, 006(041): 110-111.

[4] Zhang W, Sun F, 2019, Effect of Comprehensive Nursing Intervention on Nursing Effect and Complication Rate in Surgical Resection of Cervical Cancer. Our Health, 000(010): 274.

[5] Wang L, 2019, Observation on the Effect of Comprehensive Nursing on LEEP Knife Operation in Patients with Cervical Precancerous Lesions. World Latest Medicine Information, 19(91): 303-304.

[6] Cui X, 2020, Research on Application of Comprehensive Nursing Intervention in Patients with Concurrent Radiotherapy and Chemotherapy of Cervical Cancer. The Medical Forum, 024(009): 12411243. 